\title{
CARACTERÍSTICAS CLÍNICAS DOS PACIENTES COM HIPERTENSÃO PULMONAR EM DOIS CENTROS DE REFERÊNCIA EM SÃO PAULO
}

\author{
Monica Silveira lapa', Eloara Vieira Machado Ferreira², Carlos Jardim', Barbara do Carmo dos Santos Martins', Jaquelina Sonoe Ota Arakaki ${ }^{2}$, \\ ROGerio SOUZA ${ }^{1}$ \\ Trabalho realizado pela disciplina de Pneumologia - Grupo de Hipertensão Pulmonar - Faculdade de Medicina da Universidade de São Paulo' e \\ disciplina de Pneumologia - Grupo de Circulação Pulmonar - Universidade Federal de São Paulo²
}

\author{
*Correspondência: \\ Rogerio Souza \\ R. Afonso de Freitas, 451, \\ ap. 112 \\ São Paulo, SP \\ Cep: 04006-052 \\ rogerio.souza@incor.usp.br
}

\begin{abstract}
RESUMO
OBjetivos. Descrever as características clínicas dos pacientes com hipertensão pulmonar de dois centros de referência para tratamento de HP no pás.

Métodos. Análise retrospectiva de prontuários.

Resultados. Foram inclúídos no estudo I23 pacientes com diagnóstico de HP; 62\% destes apresentavam classe funcional III e IV (NYHA). A média da pressão sistólica do ventrículo direito (PSVD) encontrada nos pacientes estudados foi de $83,48 \pm 24,61 \mathrm{mmHg}$. Não houve correlação entre a classe funcional dos pacientes e o valor da PSVD. Cerca de 50\% dos pacientes tinham o diagnóstico de HAPI, 30\% tinham doença associada à esquistossomose e 10\% tínham HP associada a doença do tecido conectivo. $O$ tempo de dispnéia até o diagnóstico foi variável e não houve correlação com a classe funcional dos pacientes $(p>0,05)$. Apesar das diferentes etiologias, os pacientes apresentaram características semelhantes.

Conclusä̃o. Baseando-se em nossos achados, reforça-se a necessidade de investigação ativa para o diagnóstico definitivo antes da instituição de quaisquer alternativas terapêuticas para o tratamento da hipertensão pulmonar. Ressalta-se, ainda, a necessidade de um maior entendimento da hipertensão pulmonar associada à esquistossomose, em virtude da prevalência desse diagnóstico em nosso meio.
\end{abstract}

UnITERMOs: Hipertensão pulmonar. Esquistossomose. Hipertensão arterial pulmonar idiopática.

\section{INTRODUÇÃO}

A hipertensão arterial pulmonar é uma situação clínica de elevada gravidade, caracterizada pela presença de vasoconstrição pulmonar, trombose in situ e remodelamento vascular, levando à insuficiência ventricular direita progressiva e finalmente ao óbito 1,2. Define-se como hipertensão pulmonar a presença de pressão média de artéria pulmonar maior que $25 \mathrm{mmHg}$, em repouso, ou maior que $30 \mathrm{mmHg}$, ao exercício, com pressão de oclusão da artéria pulmonar menor que $15 \mathrm{mmHg} g^{3,4}$.

A hipertensão pulmonar (HP) pode se desenvolver como conseqüência de várias situações clínicas, como insuficiência ventricular esquerda, doenças do tecido conectivo, embolia pulmonar crônica, entre outras. A HP é classificada como hipertensão arterial pulmonar idiopática (HAPI) quando nenhum fator causal pode ser identificado ${ }^{4}$. Nos últimos anos, o conhecimento sobre a situação de HP aumentou de forma considerável, impulsionado pelo desenvolvimento de novas alternativas terapêuticas. Esse maior conhecimento possibilitou que fosse proposta uma classificação de forma a agrupar essas situações clínicas em categorias que apresentassem características clínicas e/ou fisiopatológicas semelhantes 5 .

Com isso, possibilitou-se também um melhor estudo epidemiológico das diversas causas de hipertensão pulmonar, assim como de suas apresentações clínicas. Dados norte-americanos e europeus descrevem a hipertensão arterial pulmonar idiopática com incidência de I a 2 casos por milhão por ano e prevalência de I 5 casos por milhão de habitantes, com predominância do sexo feminino, na razão aproximada de 2:I e início dos sintomas predominatemente após a segunda ou terceira década de vida,

Não existem, porém, quaisquer dados que caracterizem a população de pacientes portadores de hipertensão pulmonar no Brasil, o que certamente dificulta o desenvolvimento de protocolos de investigação e tratamento para nossa população, principalmente levando-se em conta características peculiares, como a presença de esquistossomose, doença endêmica em nosso meio, como causa de hipertensão pulmonar. Partindo-se dessas particularidades, recentemente foram desenvolvidas diretrizes nacionais para o diagnóstico e tratamento da hipertensão pulmonar, em que um roteiro diagnóstico adaptado foi proposto (Tabela I).

A esquistossomose é uma das doenças infecciosas mais prevalentes no mundo. É endêmica em mais de 70 países, principalmente naqueles com baixo desenvolvimento socioeconômico. O número de pessoas infectadas chega ao redor de 200 milhões, podendo atingir até $80 \%$ da população de áreas endêmicas 9.10 . A prevalência de hipertensão pulmonar associada à esquistossomose é variável; estima-se, porém, que até $30 \%$ de pacientes com esquistossomose em sua forma hepatoesplênica possam desenvolver hipertensão pulmonar ${ }^{11,13}$. 
A falta de dados epidemiológicos brasileiros em hipertensão pulmonar, associada a nossas peculiaridades, como a esquistossomose, motivou esse estudo, que tem como objetivo descrever as características dos pacientes com hipertensão arterial pulmonar de centros de referência no tratamento de hipertensão pulmonar em nosso país.

\section{População}

Foram incluídos todos os pacientes com diagnóstico de hipertensão arterial pulmonar acompanhados nos grupos de hipertensão pulmonar das disciplinas de pneumologia da Universidade de São Paulo (USP) e da Universidade Federal de São Paulo (UNIFESP), no período de janeiro de 1999 a agosto de 2003, além dos pacientes com diagnóstico de hipertensão pulmonar associada à esquistossomose. Esse período foi escolhido em função da ausência de medicações específicas para o tratamento de hipertensão pulmonar no país, mesmo sob a forma de protocolos de pesquisa, evitando-se assim o viés do encaminhamento específico para inclusão em protocolos de tratamento investigacionais.

\section{Métodos}

Ambos os centros de referência utilizam o algoritmo diagnóstico proposto pela Organização Mundial da Saúde acrescido da investigação ativa da esquistossomose ${ }^{8,14}$. Por esta razão, os dados puderam ser analisados conjuntamente. $O$ diagnóstico de hipertensão arterial pulmonar idiopática só é estabelecido após a realização de todos os exames complementares, afastando as demais condições associadas possíveis. A suspeita clínica de esquistossomose foi investigada por meio de pesquisa de ovos pesados nas fezes ou em biópsia de valva retal, além de ultrasonografia de abdome nos pacientes provenientes de áreas endêmicas de esquistossomose ${ }^{15}$.

Foi realizado o levantamento retrospectivo de todos os prontuários de 1998 a 2003, visando caracterizar as condições clínicas, laboratoriais e ecocardiográficas dos pacientes em sua apresentação nos referidos centros, com posterior correlação com o diagnóstico estabelecido após o término da investigação.

\section{Dados clínicos}

Foram recuperados os seguintes dados clínicos dos pacientes estudados: idade, sexo, o tempo de dispnéia até o diagnóstico, presença de chiado, tosse, edema de membros inferiores, dor torácica, síncope, hemoptise e dispnéia paroxística noturna. Os achados de exame físico levantados foram: presença de cianose, hepatoesplenomegalia, estase sanguínea e saturação de oxigênio em ar ambiente. De acordo com as queixas de dispnéia, os pacientes foram classificados segundo a classificação da New York Heart Association (NYHA) .

\section{Avaliação hemodinâmica}

Foram coletados os dados de pressão sistólica do ventrículo direito (PSVD), obtidos por meio da análise do jato de regurgitação tricúspide pelo ecocardiograma, realizado na avaliação inicial do paciente no centro de referência.

\section{Tabela I - Investigação diagnóstica da hipertensão pulmonar} (adaptada da ref. 8)

\begin{tabular}{|c|c|}
\hline Exame complementar & Condição associada à HP \\
\hline $\begin{array}{l}\text { Ecocardiograma } \\
\text { (microbolhas etransesofágico na } \\
\text { suspeita de shunt) }\end{array}$ & $\begin{array}{l}\text { Cardiopatia esquerda /Valvopatias } \\
\text { Cardiopatias congênitas }\end{array}$ \\
\hline $\begin{array}{l}\text { Radiografia / Tomografia de tórax } \\
\text { Espirometria }\end{array}$ & $\begin{array}{l}\text { Doença pulmonar obstrutiva crônica } \\
\text { Doençass intersticiais pulmonares } \\
\text { Anomalias de caixa torácica }\end{array}$ \\
\hline Oximetrianoturna & Distúrbios do sono \\
\hline \multicolumn{2}{|l|}{ Polissonografia } \\
\hline $\begin{array}{l}\text { Cintilografia V/Q } \\
\text { Angiotomografia de tórax }\end{array}$ & Tromboembolismo pulmonar crônico \\
\hline$\overline{F A N, F R, A N C A}$ & Doenças do tecido conectivo/vasculites \\
\hline Sorologia para HIV/VHC/VHB & Aids, hepatite B e C \\
\hline $\begin{array}{l}\text { Função hepática/PPF } \\
\text { Biópsia retal } \\
\text { Ultra-som de abdome }\end{array}$ & $\begin{array}{l}\text { Hipertensão portopulmonar } \\
\text { Esquistossomose }\end{array}$ \\
\hline
\end{tabular}

HP - hipertensão pulmonar; V/Q - ventilação e perfusão; FAN - fator antinúcleo; FR - fator reumatóide; ANCA - anticorpo anticitoplasma de neutróflo; HIV - vírus da imunodeficiência humana; VHC - vírus da hepatite C; VHB - vírus da hepatite B; Aids - síndrome da imunodeficiência humana; PPF - exame protoparasitológico de fezes

\section{Dados laboratoriais e funcionais}

Foram coletados os dados referentes à concentração média de hemoglobina (Hb); leucograma; níveis de ácido úrico (AU); e medidas espirométricas (capacidade total forçada-CVF, volume expirado forçado no primeiro segundo-VEFI).

\section{Análise estatística}

Para a comparação entre os diferentes grupos, foi utilizada a análise de variância (ANOVA) para as variáveis de distribuição normal, apresentadas como média \pm desvio padrão e o teste de Kruskal-Wallis para as variáveis não paramétricas, apresentadas como mediana (percentil 25\% - percentil 75\%), sendo considerado 0,05 como valor de significância.

\section{Resultados}

De um total de 232 pacientes com diagnóstico de hipertensão pulmonar, foram incluídos I 23 pacientes com diagnóstico de hipertensão arterial pulmonar ou HP associada à esquistossomose, excluídos os pacientes nos quais, após a realização de toda a investigação diagnóstica (Tabela I), foram evidenciadas condições associadas à hipertensão pulmonar (tromboembolismo crônico; hipertensão pulmonar associada a doenças do parênquima pulmonar; cardiopatia esquerda e distúrbios do sono) fora do contexto de hipertensão arterial pulmonar, ${ }^{8,16}$.

As características dos pacientes podem ser visualizadas na Tabela 2. No grupo estudado, existe uma predominância do sexo feminino em relação ao masculino $(2,3: 1)$ e um número maior de pacientes em classe funcional III e IV (cerca de 62\%). 
A distribuição dos diagnósticos relacionados ao desenvolvimento de hipertensão pulmonar na população estudada pode ser visualizada também na Tabela 2. Cerca de 50\% dos pacientes tinham o diagnóstico de hipertensão arterial pulmonar idiopática, 30\% de HP associada à esquistossomose, 10\% de hipertensão arterial pulmonar associada a doenças do tecido conectivo.

A associação com o uso de anorexígenos, embora pequena em relação aos demais diagnósticos (4\%), merece particular atenção em nosso meio uma vez que o uso de fenfluramina ainda ocorre, por meio de fórmulas manipuladas; isso significa que, enquanto nos países onde a família das fenfluraminas já não está mais disponível a incidência de hipertensão pulmonar associada a anorexígenos tende a diminuir, o mesmo não deve ocorrer em nosso meio, principalmente se considerarmos que o desenvolvimento de hipertensão pulmonar ocorre geralmente muito após a exposição aos anorexígenos.

A média da pressão sistólica do ventrículo direito (PSVD) em todos os pacientes estudados foi de $83,48 \pm 24,61 \mathrm{mmHg}$. Não houve associação entre os níveis de PSVD e a classe funcional dos pacientes $(p>0,05)$ (Figura I).

Ao redor de 20\% dos pacientes apresentaram achados clínicos de chiado, dispnéia paroxística noturna e cianose; mais de 40\% da população tinha tosse e edema de membros inferiores; síncope e dor torácica estavam presentes em cerca de 35\% da população.

Os achados laboratoriais globais da população estudada encontraram-se dentro dos limites da normalidade, com nível de hemoglobina de $\mid 4,6 \pm 2,8 \mathrm{~g} / \mathrm{dL}$, contagem de leucócitos de $7.552 \pm 329 \mid / \mathrm{mL}$, e o nível médio de ácido úrico foi de $6,3 \pm 2,3 \mathrm{mg} / \mathrm{dL}$.

A Tabela 3 mostra o perfil clínico dos pacientes de acordo com cada etiologia. Observa-se que a média de idades foi semelhante em todos os grupos, com uma tendência não significativa no grupo com HAP associada a anorexígenos, que apresentou a média de idade mais baixa do estudo, de 33,8 $\pm 8,4$ anos.

Em todos os grupos estudados, a maioria dos pacientes encontrava-se em classe funcional III ou IV, com exceção do grupo com HAP portopulmonar, em que a população com sintomas mais graves corresponde a $33 \%$ dos pacientes.

O tempo de dispnéia até o diagnóstico foi variável, com uma mediana geral de 20 meses, não havendo associação entre a classe funcional do paciente e o tempo de dispnéia até o diagnóstico $(p>0,05)$. A média da PSVD foi semelhante em todos os grupos $(p>0,05)$. A média da taxa de saturação de $\mathrm{O}_{2}$ não variou entre os grupos estudados $(p>0,05)$.

As medidas de CVF e VEFI foram semelhantes e dentro dos padrões da normalidade nos grupos de HAPI, DTC, HP associada à esquistossomose e anorexígenos, não evidenciando tendências claras de alteração na espirometria.

\section{Discussão}

Este estudo relata a prevalência e os achados clínicos de pacientes com HAP em dois centros brasileiros da especialidade. Não são do nosso conhecimento outras publicações nacionais relatando especificamente a prevalência de HAP no país, à exceção do projeto Queixa-

\section{Tabela 2 - Características clínicas e funcionais dos pacientes portadores de hipertensão pulmonar no período de 1999 a 2003}

\begin{tabular}{lc}
\hline Idade(anos)* & $42 \pm 14$ \\
Sexo F/M & $86 / 37$ \\
Tempo dispnéia(meses) $)^{\dagger}$ & $20(12-42)$ \\
Classe funcional I-II / III-IV & $47 / 76$ \\
Pressão sistólica de VD (mmHg)* & $83 \pm 24$ \\
Saturação O $_{2}(\%)^{*}$ & $91 \pm 12$ \\
CVF(\%)* & $89 \pm 23$ \\
VEFI (\%)* & $79 \pm 23$ \\
\hline Diagnóstico & Número de pacientes (\%) \\
HAPidiopática & $62(50,4)$ \\
Esquistossomose & $37(30,1)$ \\
DTC & $13(10,6)$ \\
Anorexígeno & $5(4,1)$ \\
Hipertensão portopulmonar & $6(4,9)$ \\
\hline
\end{tabular}

CVF(\%) - porcentagem da capacidade vital forçada em relação ao previsto

VEFI (\%) - porcentagem do volume expiratório forçado no primeiro segundo em relação ao previsto DTC - doenças do tecido conectivo

* valores apresentados como média \pm desvio padrão

${ }^{\dagger}$ valores apresentados como mediana (percentil 25\% - percentil 75\%)

\section{Figura I - Medida da pressão sistólica do ventrículo direito (PSVD) versus classe funcional (NYHA)}

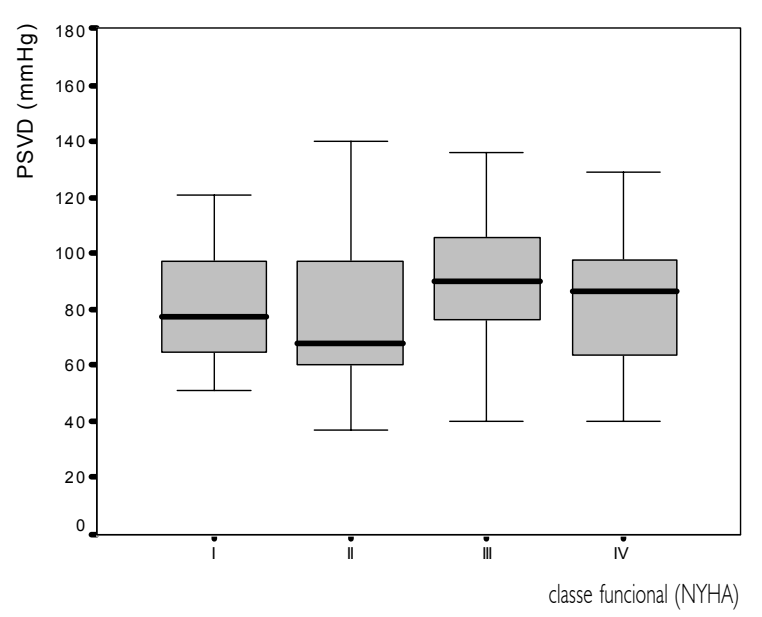

dinha, que relaciona HAP à esquistossomose endêmica' ${ }^{12}$. Supõe-se, portanto, que a amostragem atual representa a confluência dos doentes brasileiros para os grandes centros médicos, o que, embora não possa ser extrapolado para a população em geral, serve como base de dados significativa para uso nos centros de referência de tratamento de hipertensão pulmonar.

A análise mostra que os pacientes guardam características semeIhantes, apesar das diferentes etiologias. A classe funcional dos pacientes e a PSVD são semelhantes em todos os grupos. Mais de 50\% dos pacientes avaliados eram portadores de HAPI e 30\% do total tinham esquistossomose como etiologia, confirmando a esperada prevalência desta doença em nossos serviços, diferente do quadro internacional, onde praticamente se desconhece a doença. 


\begin{tabular}{|c|c|c|c|c|c|c|}
\hline & & & & & & \\
\hline & Idiopática & Esquistossomose & DTC & Anorexígeno & Portopulmonar & $\mathbf{p}$ \\
\hline Idade*(anos) & $42 \pm 15$ & $40 \pm 10$ & $50 \pm 14$ & $33 \pm 8$ & $48 \pm 18$ & 0,16 \\
\hline CF III/IV pacientes (\%) & $34(54,8)$ & $24(64,8)$ & $10(76,9)$ & $5(100)$ & $2(33,3)$ & \\
\hline Tempo dispnéia $^{\dagger}$ (meses) & $18(10-36)$ & $24(12-60)$ & $21(9-27)$ & $24(15-66)$ & $12(12-24)$ & 0,40 \\
\hline PSVD*(mmHg) & $84 \pm 25$ & $85 \pm 22$ & $79 \pm 30$ & $81 \pm 28$ & $72 \pm 16$ & 0,41 \\
\hline $\mathrm{SatO}_{2} *(\%)$ & $91 \pm 6$ & $91 \pm 20$ & $90 \pm 7$ & $93 \pm 2$ & $95 \pm 2$ & 0,20 \\
\hline
\end{tabular}

CF - classe funcional

PSVD - pressão sistólica do ventrículo direito medida pelo ecocardiograma

$\mathrm{SatO}_{2}$ - saturação arterial de oxigênio medida pela oximetria de pulso

DTC - doenças do tecido conectivo

* valores apresentados como média \pm desvio padrão

${ }^{\dagger}$ valores apresentados como mediana (percentil 25\% - percentil 75\%)

A maioria dos pacientes apresentou estadio avançado da doença, com classes funcionais III e IV e com hipertensão pulmonar grave (PSVD ao redor de $80 \mathrm{mmHg}$ ). Considerando esses dados juntamente com o tempo de dispnéia até o diagnóstico, podemos concluir que o diagnóstico de hipertensão pulmonar é feito tardiamente. A literatura estima o tempo médio para o diagnóstico em cerca de 24 meses; o tempo de dispnéia de 20 meses encontrado em nossa população indica que, apesar de todos os problemas estruturais particulares de nosso sistema de saúde, a população em seguimento nos centros de referência estudados não teve seu diagnóstico retardado em relação a centros de referência em países desenvolvidos ${ }^{3,8}$, embora ainda reflita um tempo demasiado grande para o diagnóstico, o que pode justificar a alta proporção de pacientes em classes funcionais mais avançadas.

Ainda com relação ao tempo de dispnéia, não podemos observar uma tendência ao diagnóstico mais precoce de hipertensão pulmonar em pacientes portadores de condições clínicas predisponentes ou fatores de risco tais como: doenças do tecido conectivo, hepatopatias ou, ainda, uso de anorexígenos, apesar da pesquisa ativa de hipertensão pulmonar nessas populações. Este fato leva ao questionamento sobre as ferramentas utilizadas para essa investigação, em geral apenas 0 ecocardiograma, e a periodicidade com que esses exames são realizados, especificamente nos centros de referência estudados.

\section{Conclusão}

O fato de não haver diferenças significativas nas características dos pacientes, em sua apresentação clínica, reforça a necessidade da investigação ativa dos diversos diagnósticos de hipertensão pulmonar, evitando-se assim a utilização inadequada das alternativas terapêuticas disponíveis.

Nosso estudo não evidenciou associação entre a classe funcional e o nível de pressão sistólica da artéria pulmonar, conforme já demonstrado anteriormente; esse dado vem reforçar a informação de que, embora importante para o diagnóstico de hipertensão pulmonar, o nível pressórico não é o maior determinante de gravidade dessa população; outras variáveis como o débito cardíaco, por exemplo, têm papel muito mais relevante nesse sentido, o que ressalta a necessidade da realização de medidas hemodinâmicas não limitadas ao ecocardiograma $a^{17,18}$.
O número de pacientes incluídos, embora significativo, constitui uma limitação de nosso estudo, pois não permite a extrapolação dos dados para a população geral. Mesmo assim, acreditamos que a relevância de nossos achados se baseia no fato de ser o primeiro estudo nacional que fornece informações sobre as características clínicas dos pacientes portadores de hipertensão pulmonar de nosso meio, e ainda dá a devida importância ao diagnóstico de hipertensão pulmonar relacionada à esquistossomose, cuja prevalência tão elevada justifica o desenvolvimento de pesquisas e políticas de saúde específicas para essa população.

\section{Agradecimento}

Este estudo foi possível graças ao apoio da CAPES - Ministério da Educação - Brasil

\section{Conflito de interesse: não há}

\section{SUMMARY}

Clinical CHARActeristics OF pUlmonary hypertension patients in two reference centers in the city of Sao Paulo

OBJECTIVES. Describe the clinical profile of PH patients from two pulmonary hypertensioncenters.

METHODS. Retrospective chartanalysis.

RESULTS. One hundred and twenty three PH patients were included in the study; $62 \%$ of these presented functional class III or IV (NYHA). Mean right ventricle systolic pressure (RVSP) was $83.48 \pm 24.61 \mathrm{mmHg}$. There was no correlation between functional class and RVSP. About $50 \%$ of the patients were diagnosed as IPAH; 30\% as pulmonary hypertension associated to schistosomiasis; $10 \%$ as PH associated to connective tissue diseases. Mean time of dyspnea until diagnosis was variable with no correlation with functional class $(p>0.05)$. No difference was found in the clinical presentation in spite of the diverse etiologies.

CONCLUSION. Based upon our findings, we stressed the need for an active investigation of $\mathrm{PH}$ patients prior to administration of any therapeutic alternative. We emphasized that a better understanding of $\mathrm{PH}$ related to schistosomiasis is needed due to the high prevalence of this condition among PH patients as shown in the Brazilian population. [Rev Assoc Med Bras 2006; 52(3): 139-43]

KEY WORDS: Pulmonary hypertension. Schistosomiasis. Idiopathic pulmonary hypertension. 


\section{REFERÊNCIAS}

I. Rubin LJ. Primary pulmonary hypertension. N Engl J Med 1997; 336(2): I | |-7.

2. Souza R, Bogossian HB, Humbert M, Jardim C, Rabelo C, Amato AB, et al. N-terminal-pro-brain natriuretic peptide as a haemodynamic marker in idiopathic pulmonary arterial hypertension. Eur Respir J 2005; 25(3):509-13.

3. Barst RJ, McGoon M, Torbicki A, Sibton O, Krowka MJ, Olschewski H, et al. Diagnosis and differential assessment of pulmonary arterial hypertension. J Am Coll Cardiol 2004;43( 12 Suppl S):40S-7S.

4. Channick R, Williamson TL. Diagnosis and treatment of pulmonary arterial hypertension. Cardiol Clin 2004;22(3):44I-52.

5. Strange JW, Wharton J, Phillips PG, Wilkins MR. Recent insights into the pathogenesis and therapeutics of pulmonary hypertension. Clin Sci (Lond) 2002; I02(3):253-68

6. McGoon M, Gutterman D, Steen V, Barst R, Mc Crory DC, Fortin TA, et al. Screening, early detection, and diagnosis of pulmonary arterial hypertension: ACCP evidence-based clinical practice guidelines. Chest 2004; I 26 ( I Suppl): | 4S-34S.

7. Steenhuis LH, Groen HJ, Koeter GH, Van der Mark TW. Diffusion capacity and haemodynamics in primary and chronic thromboembolic pulmonary hypertension. Eur Respir J 2000; 16(2):276-8I.

8. Souza R, Cardoso AP, Pedra CA, Jardim C, Watge D, Campos FT, et al. Diretrizes brasileiras para o manejo da hipertensão pulmonar. J Bras Pneumol 2005;3 I (2):SI-S3I.

9. Lambertucci JR, Serufo JC, Gerspacher-Lara R, Rayes AA, Teixeira R, Nobre $V$, et al. Schistosoma mansoni: assessment of morbidity before and after control. Acta Trop 2000;77( I): I 0 I-9.

10. Schwartz E. Pulmonary schistosomiasis. Clin Chest Med 2002;23 (2):433-43.
I I. Figueiredo C, Souza R, Ota JS, Moises V, Barone R, Rocha M. Pulmonary hypertension in Schistosomiasis: a chest CT study. Am J Respir Crit Care Med 2004; I 69(7):Al74.

12. LambertucciJR, Gerspacher-Lara R, Pinto-Silva RA, Barbosa MM, Teixeira $\mathrm{R}$, Barbosa HF, et al. O Projeto Queixadinha: a morbidade e o controle da esquistossomose em área endêmica no nordeste de Minas Gerais, Brasil. Rev Soc Bras Med Trop 1996;29(2): I 27-35.

13. Schwartz E, Rozenman J. Schistosomiasis. N Engl J Med 2002;347 ( 10):766-8; author reply 766-68.

14. Rubin LJ, Badesch DB. Evaluation and management of the patient with pulmonary arterial hypertension. Ann Intern Med 2005; I 43(4):282-92.

15. Richter J. Evolution of schistosomiasis-induced pathology after therapy and interruption of exposure to schistosomes: a review of ultrasonographic studies. Acta Trop 2000;77( I): I I I-3 I.

16. Simonneau G, Galie N, Rubin LJ, Langleben D, Seeger W, Domenighetti $\mathrm{D}$, et al. Clinical classification of pulmonary hypertension. J Am Coll Cardiol 2004:43(I 2 Suppl S):5S- 12 S.

17. Chemla D, Castelain V, Herve P, Lecarpentier Y, Brimioulle S. Haemodynamic evaluation of pulmonary hypertension. Eur Respir J 2002;20(5): |3|4-3|.

18. Hoeper MM, Oudiz RJ, Peacock A, Tapson VF, Haworth SG, Frost AE, et al. End points and clinical trial designs in pulmonary arterial hypertension: clinical and regulatory perspectives. J Am Coll Cardiol 2004;43( I 2 Suppl S):48S-55S.
Artigo recebido: 30/08/2005

Aceito para publicação: 27//2/2005 